\title{
Aedes albopictus (S) na região de São José do Rio Preto, SP: estudo da sua infestação em áreajá ocupada pelo Aedes aegypti ediscussão de seu papel como possível vetor de dengue e febre amarela
}

\author{
Aedesalbopictus (S) in the region of São José do Rio Preto, SP, Brazil: a study of its infestation \\ in an area where Aedesaegypti was already established and a discussion \\ of its role as a possible vector of dengue and yellow fever
}

\author{
Francisco Chiaravalloti Neto ${ }^{1,2}$, Margareth Regina Dibo1, Angelita Anália \\ Carniel Barbosa' e Marcos Battigaglia'
}

\begin{abstract}
Resumo Os objetivos deste trabalho foram estudar a evolução da infestação pelo Aedes albopictus na região de São José do Rio Preto, área já ocupada pelo Aedes aegypti e discutir seu papel na transmissão de doenças. Com informações obtidas em medidas de densidade larvária realizadas em áreas urbanas dos municípios, analisaram-se: ano e local de ocorrência, composição e localização das amostras larvárias; recipientes e Índices de Breteau. Até maio de 2001, o vetor já se encontrava em 96 dos 100 municípios da região. O Aedes albopictus, comparativamente ao Aedes aegypti, ocupou em maior proporção o peridomicílio e apresentou maior grau de associação com recipientes naturais e descartáveis. O comportamento endêmico do dengue, a ocorrência de casos autóctones de febre amarela silvestre na região e a reconhecida competência do vetor para estas doenças implicam em considerar a possibilidade de sua participação na transmissão do dengue $e$ na reurbanização da febre amarela.
\end{abstract}

Palavras-chaves: Aedes albopictus. Infestação. Dengue. Febre amarela.

\begin{abstract}
The objectives of this survey were to study the evolution of Aedes albopictus infestation in São José do Rio Preto region, SP, an area already occupied by Aedes aegypti and also to discuss its role in transmitting diseases. Analyzing data from urban mosquito larval density surveys of the region's municipalities, year and site of the occurrence, composition and location of larval samples, breeding containers and Breteau indices were studied. By May 2001, the vector was found in 96 of 100 municipalities. Aedes albopictus compared with Aedes aegypti was found in greater proportions close to dwellings and presented greater degrees of association in natural and discarded containers. Endemic behavior of dengue, occurrences of local cases of sylvatic yellow fever and recognized competence of the vector's transmission of these diseases suggest the necessity to consider its possible participation in the transmission of dengue and the re-urbanization of yellow fever.
\end{abstract}

Key-words: Aedes albopictus. Infestation. Dengue. Yellow fever.

O Aedes albopictus, que se acredita ser originário da Ásia ${ }^{9}$, apresenta elevada capacidade de dispersão ${ }^{10}$ associada à colonização tanto de recipientes naturais como artificiais com água ${ }^{18}$, e em áreas urbanas é um mosquito com comportamento semidoméstico ${ }^{14}$. Temse observado que, durante cada ciclo gonotrófico, a fêmea deste vetor exerce a hematofagia em várias oportunidades e pode fazê-lo em uma ampla variedade de animais, mamíferos e aves, sendo ainda altamente antropofílica, o que aumenta sua capacidade de transmitir enfermidades em diferentes hospedeiros ${ }^{22}$.Também na
Ásia é vetor primário de dengue principalmente em áreas rurais e secundário em outras províncias desta região, junto com o Aedes aegypti ${ }^{16}$. A transmissão do vírus pode ser horizontal ou vertical, tendo esta última importância epidemiológica pela possibilidade de estabelecer novos focos de dengue com a importação de materiais que contenham ovos infectados ${ }^{22}$. Até o momento, na América, o mosquito não foi implicado na transmissão de dengue, mas no Brasil, vírus dengue Tipo 1 foram isolados de dois pools de larvas coletadas na cidade de Campos Altos, Estado de Minas Gerais ${ }^{20}$.

\footnotetext{
1. Superintendência de Controle de Endemias. 2. Faculdade de Medicina de São José do Rio Preto, São José do Rio Preto, SP. Endereço para correspondência: Dr. Francisco Chiaravalloti Neto. R. Rio Negro 160, 15091-390 São José do Rio Preto, SP Tel.: 55 17-224-5522, Fax: 5517 224-9888, e-mail: fcneto@famerp.br

Recebido para publicação em 13/8/2001.
} 
Estudos de competência vetorial de diferentes linhagens de Aedes albopictus introduzidos nas Américas, indicam que esta espécie é um competente vetor para a febre amarela ${ }^{19}$. Pela sua ampla valência ecológica, adaptando-se facilmente aos ambientes rural, urbano e peri-urbano, presume-se que possa servir de ponte entre os ciclos silvestre e urbano da febre amarela $a^{117}$. Outras doenças como Mayaro e Oropouche, vírus causadores de Encephalites e a filária Dirofilaria immitis podem eventualmente ser transmitidas pelo Aedes albopictus ${ }^{8222}$.

O Aedes albopictus foi encontrado pela primeira vez no Brasil em 1985 no Estado do Rio de Janeiro e no Estado de São Paulo em 1986². A infestação do Estado de São Paulo por essa espécie seguiu o sentido leste-oeste ${ }^{3}$.

Dadas as características biológicas do Aedes albopictus, como a sua capacidade de se alimentar, entre outros, de sangue humano, a ocupação de ambientes rurais, urbanos e peri-urbanos e de sua competência vetorial para transmitir várias doenças, principalmente dengue e febre amarela, é importante que se acompanhe a evolução da sua presença e sua convivência com $o$ Aedes aegypti no Estado de São Paulo. Na região de São José do Rio Preto, o estudo do comportamento da espécie se torna necessário uma vez que o dengue vem assumindo características de transmissão endêmica ${ }^{5} \mathrm{e}$ a região encontra-se em área epizoótica de transmissão de febre amarela silvestre ${ }^{11}$. O estudo de dados secundários, coletados nas atividades desenvolvidas no Programa de Controle de Vetores do Dengue e da Febre Amarela do Estado de São Paulo, pode fornecer indicações para essas questões e gerar novas hipóteses.

$\mathrm{O}$ presente trabalho tem por objetivos descrever e analisar a evolução da infestação pelo Aedes albopictus na região de São José do Rio Preto e discutir seus possíveis papéis na transmissão do dengue e como elo de ligação entre a formas silvestre e urbana da febre amarela.

\section{MATERIAL E MÉTODOS}

$A$ área de estudo corresponde à região de São José do Rio Preto situada a noroeste do Estado de São Paulo, na divisa com Minas Gerais e Mato Grosso do Sul. É constituída por 100 municípios, agrupados nas subregiões de Catanduva, São José do Rio Preto, Votuporanga e Jales (Figura 1). Corresponde na estrutura da Secretaria Estadual de Saúde à Direção Regional XXII (DIR XXII) e ao Serviço Regional 8 da
Superintendência de Controle de Endemias (SUCEN). Toda a região encontra-se na área epizoótica de transmissão de febre amarela silvestre ${ }^{11}$. Em 1985, o Aedes aegypti foi detectado na região ${ }^{21}$ e até 1993 já infestava todos os seus municípios (SUCEN: dados não publicados). O Aedes albopictus foi encontrado pela primeira vez na região em 1991 e até 1994 já infestava 35 dos seus municípios ${ }^{4}$.

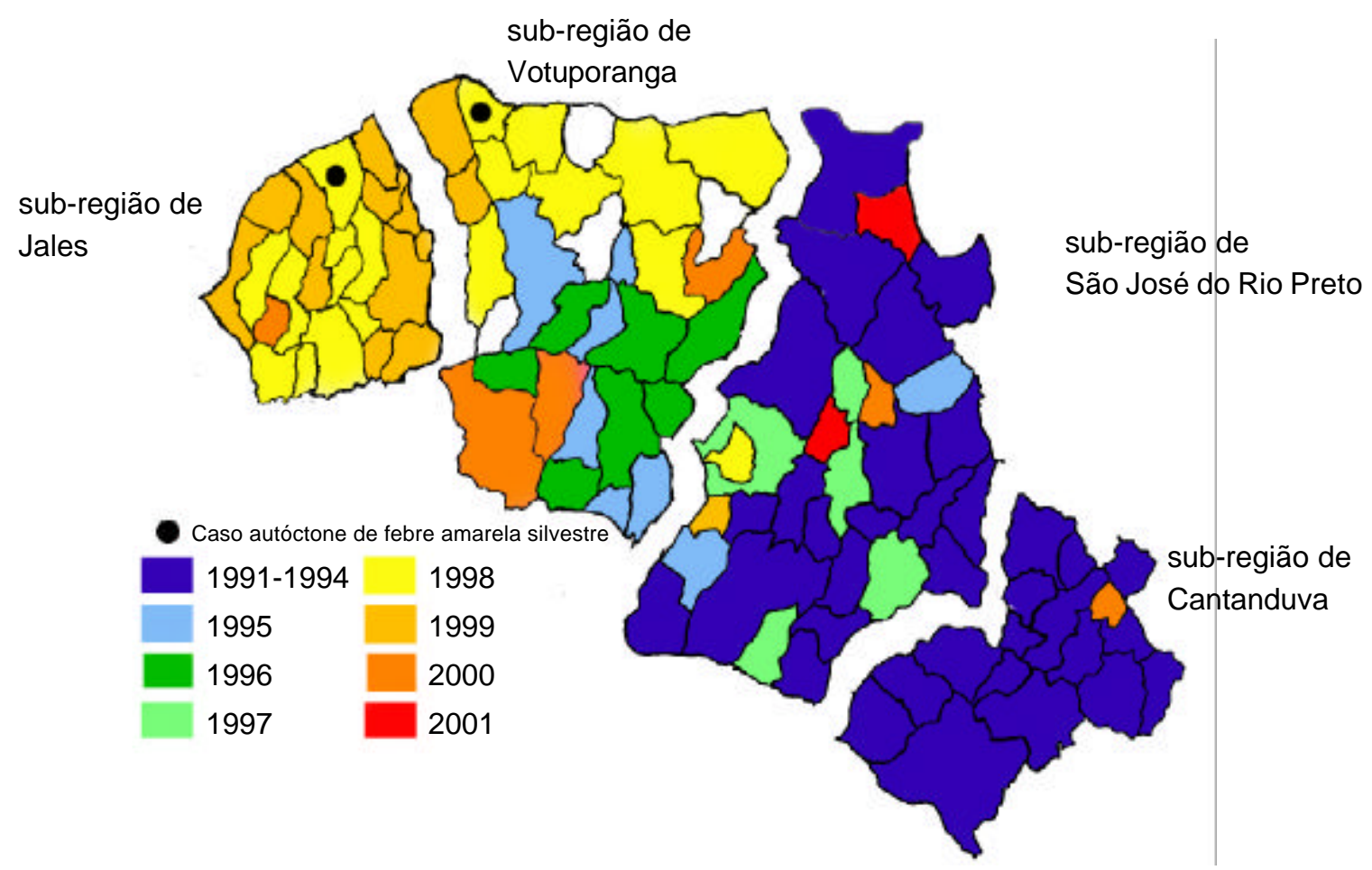

Figura 1 - Municípios segundo sub-regiões, ocorrência e casos autóctones de febre amarela silvestre e ano do primeiro encontro de larvas de Aedes albopictus, região de São José do Rio Preto, SP, 1981 a 2001. 
A SUCEN realiza anualmente, no período mais favorável aos vetores, ao menos uma medida de densidade larvária, por amostragem de quadras e residências ${ }^{1}$, em cada um dos municípios da região. Nessas medidas os funcionários visitam as casas das quadras sorteadas, pesquisam recipientes existentes e coletam larvas de mosquitos, quando presentes, obtendo as denominadas amostras larvárias. Essas amostras são encaminhadas aos laboratórios para identificação e contagem, sendo consideradas positivas aquelas contendo larvas de Aedes aegypti e/ou Aedes albopictus. Os dados são registrados em boletins específicos e a principal medida de densidade larvária obtida é o Índice de Breteau (IB) para cada uma das espécies vetoras. O IB é calculado dividindo-se o número de recipientes com larvas para determinada espécie pelo número de casas inspecionadas, com a multiplicação do resultado por $100^{13}$.

A partir dos dados disponíveis nesses boletins, considerando-se o período de 1995 a 2001 e utilizandose as informações apenas a partir do mês de detecção da infestação por Aedes albopictus em cada um dos municípios, constituíram-se dois bancos de dados. No primeiro registraram-se, para cada amostra larvária positiva, o município, a composição (somente Aedes albopictus, somente Aedes aegyptiou ambas espécies), a localização nos imóveis (intradomicílio ou peridomicílio), os tipos de recipientes. No outro banco registaram-se, para cada medida de densidade larvária, os valores do IB para Aedes aegypti e do IB para Aedes albopictus. Estes bancos foram criados no Programa Excel. As freqüências simples de cada variável, os cruzamentos entre elas e as análises estatísticas foram realizadas utilizando-se os Programas Excel, Epi Info e Stata.

O estudo da ocupação dos recipientes pelos vetores baseou-se em classificação presente nos boletins de densidade larvária utilizados pela SUCEN no período de 1995 a 1997. A partir de 1998 essa categorização sofreu algumas alterações. Os recipientes concordantes ou aqueles especificados nos boletins foram reclassificados segundo a subdivisão anterior e os sem especificação foram desconsiderados.

Com as informações disponíveis e com aquelas provenientes de estudo já realizado 4 obteve-se, a partir de 1991 até 2001, a ocupação dos municípios da região pelo Aedes albopictus.

Para verificação da existência de associação entre composição das amostras larvárias e sua localização nos imóveis, entre composição e sub-regiões e entre composição e tipos de recipientes ocupados foi utilizado o teste estatístico do Qui-quadrado para independência. Esta última relação foi analisada também através do método estatístico denominado Análise de Dependência (Anadep) ${ }^{7}$. Analisou-se a distribuição dos IB segundo as duas espécies e sub-regiões a partir da apresentação dos boxplots, onde pode-se visualizar os valores dos percentis $25\left(p_{25}\right), 50\left(p_{50}\right)$ e $75\left(p_{75}\right)$, os limites inferior $\left(p_{25}-1,5 .\left(p_{75}-\right.\right.$ $\left.\left.p_{25}\right)\right)$ e superior $\left(x_{75}+1,5 .\left(p_{75}-p_{25}\right)\right)$ e os valores acima ou abaixo destes limites, considerados pontos anômalos ${ }^{6}$.

Informações sobre a ocorrência de casos autóctones de febre amarela silvestre na região foram obtidas a partir da consulta às fichas individuais de notificação do Sistema de Informações de Agravos de Notificação ( SINAN ) do Sistema Único de Saúde (SUS).

\section{RESULTADOS}

No período de 1995 a 2000, foram obtidas, nos levantamentos realizados para a medida de IB, 7142 amostras larvárias contendo exemplares de Aedes aegypti e/ou Aedes albopictus, em municípios infestados por ambas as espécies. A identificação específica revelou que $89,8 \%$ dessas amostras continham apenas Aedes aegypti, 6,1\% apenas Aedes albopictus e 4,1\% ambas as espécies. São apresentados, para o mesmo período, a distribuição das amostras larvárias por espécie(s) encontrada(s) segundo localização nos domicílios (Tabela 1) e segundo tipos de recipientes (Tabela 2). Esta última relação é também apresentada na Figura 2, utilizando-se a técnica estatística denominada Análise de Dependência ${ }^{7}$.

Tabela 1 - Amostras larvárias segundo localização no domicílio e espécie(s) encontrada(s), região de São José do Rio Preto, 1995 a $2000^{*}$.

\begin{tabular}{|c|c|c|c|c|c|c|c|}
\hline \multirow[b]{3}{*}{ Localização } & \multicolumn{6}{|c|}{ Espécie encontrada } & \multirow{3}{*}{ Total } \\
\hline & \multicolumn{2}{|c|}{ Aedes albopictus } & \multicolumn{2}{|c|}{ Aedes aegypti } & \multicolumn{2}{|c|}{ ambas } & \\
\hline & $\mathrm{n}^{0}$ & $\%$ & $\mathrm{n}^{\circ}$ & $\%$ & $\mathrm{n}^{0}$ & $\%$ & \\
\hline Intradomicílio & 14 & 3,2 & 505 & 7,9 & 9 & 3,1 & 528 \\
\hline Peridomicílio & 420 & 96,8 & 5.909 & 92,1 & 285 & 96,9 & 6.614 \\
\hline Total & 434 & 100,0 & 6.414 & 100,0 & 294 & 100,0 & 7.142 \\
\hline
\end{tabular}

No período de 1995 a 2001, detectou-se a presença de Aedes albopictus em 61 municípios, além dos 35 anteriormente infestados ${ }^{4}$, totalizando 96 municípios com o vetor. Apresentamos na Figura 1, para o período de 1991 a 2001, a evolução da infestação da região por Aedes albopictus. Na sub-região de Catanduva, em 16 dos seus 17 municípios, detectou-se a presença do vetor entre 1991 e 1994 (3 anos); na sub-região de São José do Rio Preto a infestação foi detectada entre 1992 e 2001 (10 anos); em 26 dos 30 municípios da 
Tabela 2 - Amostras larvárias segundo espécie(s) encontrada(s) e tipo de recipiente, região de São José do Rio Preto, 1995 a $2000 *$

\begin{tabular}{|c|c|c|c|c|c|c|c|c|}
\hline \multirow[b]{3}{*}{ Tipo de recipiente } & \multicolumn{6}{|c|}{ Espécie encontrada } & \multirow{2}{*}{\multicolumn{2}{|c|}{ Total }} \\
\hline & \multicolumn{2}{|c|}{ Aedes albopictus } & \multicolumn{2}{|c|}{ Aedes aegypti } & \multicolumn{2}{|c|}{ ambas } & & \\
\hline & $\mathrm{n}^{\circ}$ & $\%$ & $\mathrm{n}^{\circ}$ & $\%$ & $\mathrm{n}^{\circ}$ & $\%$ & $\mathrm{n}^{0}$ & $\%$ \\
\hline Recipientes para armazenamento de água & 17 & 2,7 & 584 & 93,7 & 22 & 3,5 & 623 & 100,0 \\
\hline Pneus & 19 & 3,4 & 526 & 93,1 & 20 & 3,5 & 565 & 100,0 \\
\hline Vasos e pratos de plantas & 92 & 4,0 & 2.134 & 92,5 & 82 & 3,5 & 2.308 & 100,0 \\
\hline Bebedouros animais & 19 & 4,3 & 401 & 90,5 & 23 & 5,2 & 443 & 100,0 \\
\hline Recipientes fixos & 3 & 5,2 & 53 & 91,4 & 2 & 3,4 & 58 & 100,0 \\
\hline Outros recipientes removíveis & 59 & 7,5 & 684 & 86,9 & 44 & 5,6 & 787 & 100,0 \\
\hline Latas, potes, frascos e copos & 142 & 7,6 & 1.649 & 88,4 & 75 & 4,0 & 1.866 & 100,0 \\
\hline Naturais & 5 & 17,9 & 22 & 78,6 & 1 & 3,6 & 28 & 100,0 \\
\hline
\end{tabular}

${ }^{*}$ Qui-quadrado $=67,035 ; p<0,0001$

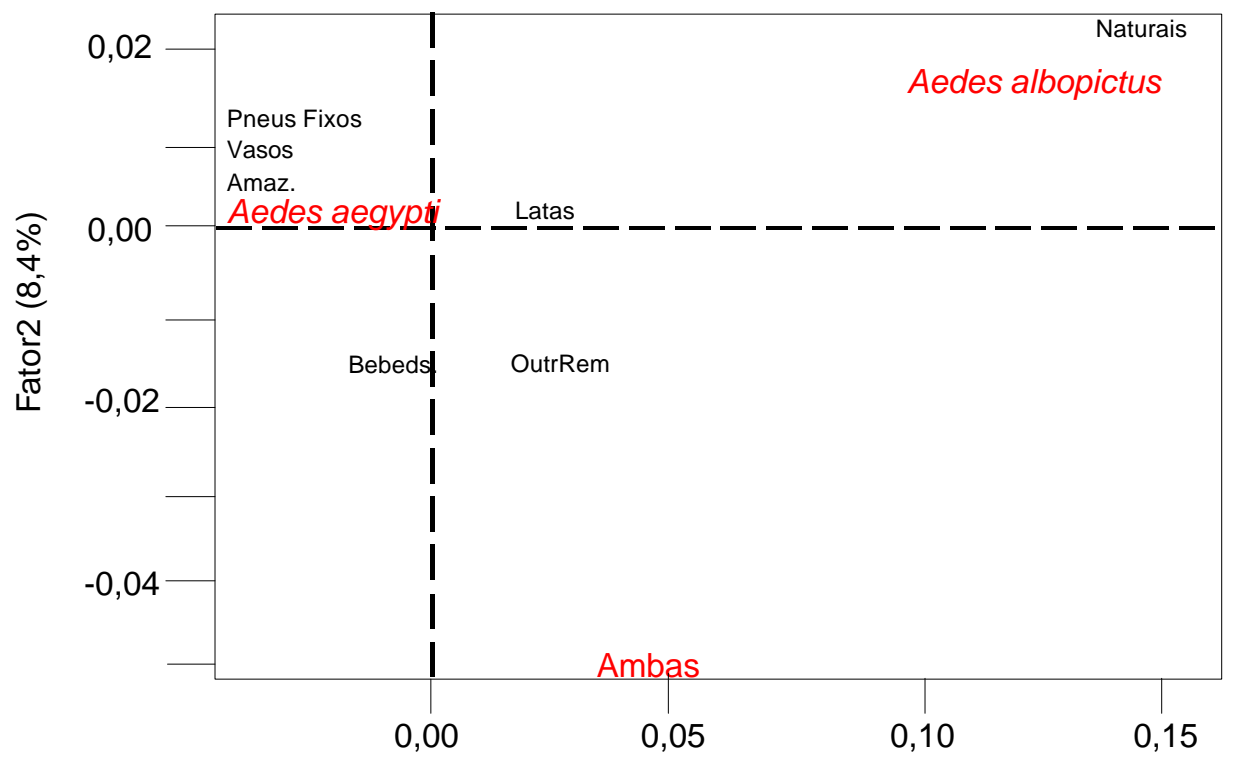

Fator1 (91,5\%)

Figura 2 - Análise de dependência (Anadep): amostras larvárias segundo espécie(s) encontrada(s) e tipo recipiente, região de São José do Rio Preto, 1995 a 2000.

sub-região de Votuporanga a infestação foi descoberta entre 1995 e 2001 (7 anos), existindo 4 onde o vetor ainda não foi identificado; na sub-região de Jales detectou-se a presença do vetor em todos os seus municípios no período de 1998 a 2000 (3 anos).

$\mathrm{Na}$ Tabela 3, apresenta-se a distribuição das amostras larvárias segundo a espécie encontrada e as áreas da região. Na Figura 3, apresentam-se os boxplots dos IB para Aedes albopictus segundo as quatro áreas da região de São José do Rio Preto.
No ano de 2000, ocorreram dois casos autóctones de febre amarela silvestre em área epizoótica na região (Figura 1) ${ }^{11}$. O primeiro caso, notificado em março, ocorreu em Santa Albertina, e o segundo, notificado em abril, em Ouroeste. Os locais de ocorrência de transmissão pertencem à zona rural destes municípios e se caracterizam como pontos de pesca e áreas de matas situados nas margens do Rio Grande fazendo divisa com o Estado de Minas Gerais. Nas áreas urbanas desses municípios, detectou-se a presença de Aedes albopictus em 1998 durante a realização de medidas de densidade larvária.

\section{DISCUSSÃO}

A grande proporção de amostras com Aedes albopictus encontrada fora dos domicílios (em torno de $97 \%$ ) foi ainda inferior à encontrada em levantamento realizado entre 1991 e 19944. Esta maior preferência pelo ambiente peridomiciliar é concordante com a literatura onde se tem ressaltado o seu maior grau de exofilia e 
Tabela 3 - Amostras larvárias segundo sub-regiões e espécie(s) encontrada(s), região de São José do Rio Preto, 1995 a $2000^{*}$.

\begin{tabular}{|c|c|c|c|c|c|c|c|c|}
\hline \multirow[b]{3}{*}{ Sub-regiões } & \multicolumn{6}{|c|}{ Espécie encontrada } & & \\
\hline & \multicolumn{2}{|c|}{ Aedes albopictus } & \multicolumn{2}{|c|}{ Aedes aegypti } & \multicolumn{2}{|c|}{ Ambas } & \multicolumn{2}{|c|}{ Total } \\
\hline & $\mathrm{n}^{\mathrm{O}}$ & $\%$ & $\mathrm{n}^{\circ}$ & $\%$ & $\mathrm{n}^{\circ}$ & $\%$ & $\mathrm{n}^{\circ}$ & $\%$ \\
\hline Catanduva & 28 & 1,0 & 2.807 & 95,8 & 93 & 3,2 & 2.928 & 100,0 \\
\hline São José do Rio Preto & 51 & 3,3 & 1.400 & 90,7 & 92 & 6,0 & 1.543 & 100,0 \\
\hline Votuporanga & 74 & 3,9 & 1.755 & 91,3 & 93 & 4,8 & 1.922 & 100,0 \\
\hline Jales & 281 & 37,5 & 452 & 60,4 & 16 & 2,1 & 749 & 100,0 \\
\hline Total & 434 & 6,1 & 6.414 & 89,8 & 294 & 4,1 & 7.142 & 100,0 \\
\hline
\end{tabular}

${ }^{*}$ Qui-quadrado $=1493,5 ; p<0,0001$

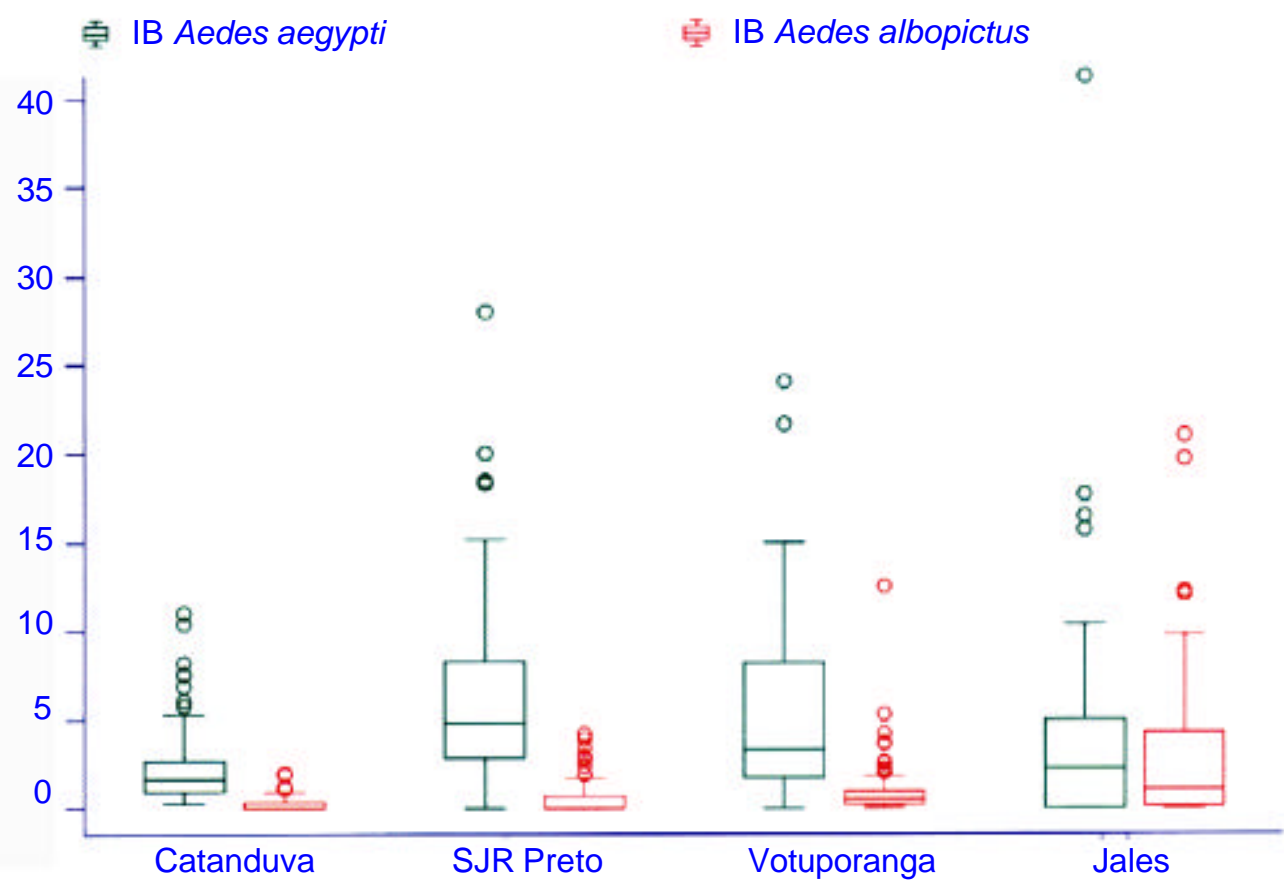

Figura 3 - Boxplots dos IB para Aedes aegypti e Aedes albopictus segundo sub-regiões, região de Săo José do Rio Preto, 1995 a 2000.

seus hábitos semidomésticos 81415 . Enquanto o pequeno grau de ocupação do intradomicílio pelo Aedes albopictus está relacionado com as suas características biológicas e ecológicas ${ }^{914}$, a diminuição do encontro de Aedes aegypti em relação ao período de 1991 a 1994 , passando de $17,5 \%$ para $7,9 \%{ }^{4}$, pode ser explicada por uma diminuição da oferta de recipientes neste ambiente (SUCEN: dados não publicados).

As duas espécies ocuparam os recipientes de modos diversos, observando-se na Figura 2 um maior grau de associação entre o Ae. albopictus com os criadouros naturais e os materiais descartáveis (latas, potes, frascos, copos, e outros tipos de recipientes removíveis); e entre o Aedes aegypti e os recipientes para armazenamento de água, vasos e pratos de plantas, recipientes fixos (ralos, privadas, caixas de descarga, piscinas, etc), bebedouros e pneus.
O fator 1 , com 91,9\% da variabilidade total, pode representar o grau de sinantropia de cada uma das espécies de vetores, e também a distância média de cada um dos tipos de recipientes ao intradomicílio, considerando-o como o centro da moradia. Os recipientes mais relacionados com o Aedes aegypti podem ser aqueles mais próximos deste centro (com exceção dos pneus), e os mais associados ao Aedes albopictus, os mais distantes.

Vasos e pratos de plantas, recipientes para armazenamento de água, recipientes fixos e bebedouros (principalmente de cães e gatos) estão localizados no intradomicílio ou nas suas imediações. Os recipientes naturais e os descartáveis se posicionam, em média, em locais mais afastados, e o Aedes albopictus, com comportamento semi-doméstico, acaba por ocupá-los. $\mathrm{O}$ auto grau de associação entre Aedes albopictus e os 
recipientes naturais está relacionado também à sua preferência por este tipo de criadouro ${ }^{1415}$.

Além de estar presente de forma diferenciada no ambiente doméstico, o Aedes albopictus deve ocupar as áreas urbanas dos municípios de modo não uniforme, provavelmente sendo mais freqüente nas áreas periféricas e com maior presença de vegetação. Estas questões apresentam relevância e merecem avaliação. E interessante realizar estudos espaciais para caracterizar como ocorre a ocupação dos ambientes doméstico e urbano pelo vetor.

A ocupação da região e suas subdivisões pelo Aedes albopictus ocorreu em momentos e velocidades diferentes. A área de Catanduva foi ocupada mais precocemente e a de Jales mais tardiamente, de modo concordante com o sentido de ocupação do Estado de São Paulo, de leste para oeste ${ }^{3}$. Nestas sub-regiões a velocidade em que ocorreu o encontro do vetor foi bem maior do que nas outras duas áreas, principalmente na de Jales, onde em apenas 2 anos (1998 e 1999) a infestação foi identificada em $96 \%$ dos seus municípios.

Esta velocidade de ocupação diferenciada deve estar relacionada, entre outros motivos, à pressão exercida por áreas vizinhas. No caso da área de Catanduva, a pressão foi exercida por municípios já infestados das DIR IX (Barretos), DIR VII (Araraquara) e DIR VIII $\left(\right.$ Bauru) ${ }^{12}$. A rápida ocupação da área de Jales está relacionada à pressão exercida por municípios já infestados da própria região de São José do Rio Preto, da DIR VI (Araçatuba) (Pauliquevis Jr C: dados não publicados) e do Estado de Minas Gerais (Fundação Nacional de Saúde: dados não publicados).

A área de Jales, além da rápida ocupação pelo vetor, tem como característica uma maior freqüência de ocorrências. Nas outras áreas o Aedes albopictus foi encontrado (sozinho ou acompanhado pelo Ae. aegypti) em proporções variando entre 4,2\% (sub-região de São José do Rio Preto) e 9,3\% (sub-região de Catanduva) do total das amostras, valores semelhantes aos encontrados no levantamento realizado entre $1991 \mathrm{e}$ 1994 (4,7\%), onde o vetor ocupava as áreas de Catanduva e parcialmente a de São José do Rio Preto ${ }^{4}$ $\mathrm{Na}$ área de Jales, a proporção das amostras com o vetor atingiu quase $40 \%$, valor bem superior ao das outras áreas e ao encontrado para período de 1991 a 1994. Chama atenção que a menor proporção de encontro de ambas as espécies em toda a região se dê nesta área $(2,1 \%)$, sugerindo que elas possam estar presentes em locais distintos das áreas urbanas de seus municípios.
Este comportamento diferenciado do Aedes albopictus é confirmado comparando-se os boxplots dos IB para esta espécie, notando-se maior amplitude de variação dos IB da área de Jales em relação às outras três. Comparando-se os boxplots das duas espécies vetoras verifica-se menor amplitude de variação dos IB para Aedes albopictus nas áreas de Catanduva, São José do Rio Preto e Votuporanga em relação aos IB para Aedes aegypti. No caso da área de Jales as distribuições dos valores dos IB para as duas espécies são semelhantes. A rápida ocupação pelo vetor e a maior expressividade dos IB nesta área deve estar relacionada, além da pressão exercida por áreas vizinhas já infestadas, com a existência de melhores condições para o desenvolvimento da espécie, cabendo-se investigar quais são elas.

A ocupação de quase todas as áreas urbanas da região pelo $A e$. albopictus somada às suas características semidomésticas e silvestres e à sua ampla valência ecológica ${ }^{14}$ levam a crer que ele possa estar presente em áreas rurais e de matas residuais. Estes fatos analisados em conjunto com a sua reconhecida competência vetorial em transmitir o vírus da febre amarela ${ }^{19} \mathrm{e}$ do dengue ${ }^{1622}$ implicam em considerar como possível a sua participação no ciclo destas doenças, principalmente na área de Jales, onde o vetor aparece com maior intensidade.

A ocorrência de casos autóctones de febre amarela silvestre em área epizoótica pertencente à região coloca como prioritária a análise do risco de sua reurbanização no estado de São Paulo, com o Aedes albopictus podendo servir de elo de ligação entre as formas silvestre e urbana da doença. Além do acompanhamento da situação do vetor nas áreas urbanas, é importante pesquisar pela sua presença nas áreas rurais e de matas residuais, intensificar a vigilância epidemiológica para a rápida detecção de casos e manter a população da região vacinada contra a doença.

No caso do dengue, os argumentos expostos acima junto com o caráter endêmico que a doença vem apresentando na região de São José do Rio Preto ${ }^{5}$ criam condições teóricas para que o vetor possa transmitir a doença, de maneira semelhante ao que ocorre na Ásia ${ }^{16}$. Há a necessidade de realização de pesquisas procurando identificar a presença do vírus no vetor, mantendo-se a atenção para a identificação de situações onde a transmissão possa estar estritamente a ele relacionada.

\section{AGRADECIMENTOS}

Agradecemos a Beatriz A.C. Belini, Marcelo Donizete Papa, Neusa F.A. Santana e Perpétua M. Magalhães pela colaboração no levantamento dos dados e a Cláudio Martinez e Luis Henrique Oliveira Ferreira pela colaboração na confecção das ilustrações. 


\section{REFERÊNCIAS BIBLIOGRÁFICAS}

1. Alves MCP, Gurgel SM, Almeida MCRR. Plano amostral para cálculo de densidade larvária de Aedes aegypti e Aedes albopictus no Estado de São Paulo, Brasil. Revista de Saúde Pública 25: 251-256, 1991.

2. Brito M, Marques GRAM, Marques CCA, Tubaki RM. Primeiro encontro de Aedes (Stegomyia) albopicuts (Skuse) no estado de São Paulo (Brasil). Revista de Saúde Pública 20:489, 1986.

3. Burali GM, Marques GRAM, Gerola Jr O. Disribuição geográfica de Aedes aegypti e Aedes albopictus no Estado de São Paulo. Revista da Sociedade Brasileira de Medicina Tropical 24 (supl II): 182, 1991

4. Chiaravalloti-Neto F. Descrição da colonização de Aedes albopictus (Diptera: Culicidae) na região de São José do Rio Preto, SP, 1991-1994. Revista da Sociedade Brasileira de Medicina Tropical 29: 543-548, 1996.

5. Chiaravalloti-Neto F. Epidemiologia da dengue nas regiões de São José do Rio Preto e Araçatuba, São Paulo, 1990 a 1996. Tese de Doutorado, Faculdade de Saúde Pública da Universidade de São Paulo, São Paulo, SP, 1999.

6. Cleveland WS. Visualizing data. Hobart Press, Summit, 1993.

7. Cordeiro JA. Análise de dependência: uma técnica para estudo de tabelas cruzadas. Tese de Livre Docência, Instituto de Biociências, Letras e Ciências Exatas da Universidade Estadual Paulista, São José do Rio Preto, SP, 1990.

8. Estrada-Franco JG, Craig JR GB. Biology, disease relationships, and control of Aedes albopictus. Technical Paper № 42. Pan American Health Organization, Washington, 1995.

9. Forattini OP. Identificação de Aedes (Stegomya) albopictus (Skuse) no Brasil. Revista de Saúde Pública 20: 5, 1986.

10. Forattini OP. Produtividade de criadouro de Aedes albopictus em ambiente urbano. Revista de Saúde Pública 31:545-555, 1997.

11. Fundação Nacional de Saúde. Manual de vigilância epidemiológica de febre amarela. Ministério da Saúde, Brasília, 1999.

12. Glasser CM. Estudo da infestação do estado de São Paulo por Aedes aegypti e Aedes albopictus. Dissertação de Mestrado, Faculdade de Saúde Pública da Universidade de São Paulo, São Paulo, SP, 1997.
13. Gomes AC. Medidas dos níveis de infestação urbana para Aedes (Stegomyia) aegypti e Aedes (Stegomyia) albopictus em programas de vigilância entomológica. Informe Epidemiológico do SUS 7: 49-57, 1998.

14. Hasanuddin I, Miyagi I, Toma T, Kamimura K. Breeding habitats of Aedes aegypti (L) and Aedes albopictus (Skuse) in villages of Barru, South Sulawesi, Indonesia. Southeast Asian Journal of Tropical Medicine and Public Health 28: 844-850, 1997.

15. Hawley WA. The biology of Aedes albopictus. Journal of the American Mosquito Control Association 4: 1-39, 1988.

16. Marques CCA, Marques GRA, Degallier N. Is Aedes albopictus only a pest mosquito or also a vector of arboviruses in Brazil? In: Rosa APAT, Vasconcelos PFC, Rosa JFST (eds) An overview of arbovirology in Brazil and neighbouring countries. Evandro Chagas Institute, Belém, p. 248-260, 1998.

17. Monath TP. Yellow fever: A medically neglected disease. Report on a seminar. Reviews on Infectious Diseases 9: 165-175, 1987.

18. Moore CG, Francy DB, Eliason DA, Monath TP. Aedes albopictus in the United States: rapid spread of a potential disease vector. Journal of the American Mosquito Control Association 4: 356361, 1988.

19. Savage HM, Ezike VI, Nwankwo ACN, Spiegel R, Miller BR. First record of breeding populations of Aedes albopictus in Continental Africa: implications for arboviral transmission. Journal of the American Mosquito Control Association 8: 101-103, 1992.

20. Serufo JC, Oca HM, Tavares VA, Souza AM, Rosa RV, Jamal MC, Lemos JR, Oliveira MA, Nogueira RMR, Schatzmayr HG. Isolation of dengue virus type 1 from larvae of Aedes albopictus in Campos Altos City, state of Minas Gerais, Brazil. Memória do Instituto Oswaldo Cruz 88: 503-504, 1993.

21. Superintendência de Controle de Endemias. Plano de emergência para o controle de vetores do dengue e da febre amarela no verão de 1991/1992. Secretaria de Estado da Saúde, São Paulo, 1991.

22. Vélez ID, Quiñones ML, Suárez M, Olano V, Murcia LM, Correa E, Arévalo C, Pérez L, Brochero $H$, Morales A. Presencia de Aedes albopictus en Leticia, Amazonas, Colombia. Biomédica 18: 192-198, 1998. 